\title{
85th Annual Meeting of the Swiss Society for Dermatology and Venereology
}

September 18-20, 2003, Lausanne

Editor: Prof. R.G. Panizzon

\section{Contents}

Use of Duplex Ultrasonography in the Treatment of

Thromboangiitis obliterans with lloprost

B. Noël, R.G. Panizzon

Dermoscopy for the in vivo Detection of Sarcoptes scabiei

C. Prins, L. Stucki, L. French,

J.-H. Saurat, R.P. Braun

\section{First Report of Arthroderma} benhamiae in Switzerland

J. Fumeaux, M. Mock, B. Ninet, I. Jan,

O. Bontems, B. Léchenne, D. Lew,

R.G. Panizzon, O. Jousson, M. Monod

Progressive Respiratory Failure in Paraneoplastic Pemphigus Associated with Chronic Lymphocytic Leukemia

M. Brönnimann, U. von Felbert,

M. Streit, T. Hunziker, L.R. Braathen

Micronodular Kaposi's

Sarcoma - A New Variant of Classic-Sporadic Kaposi's

Sarcoma

W. Kempf, G. Cathomas, G. Burg,

R.M. Trüeb . . . . . . . . . . 255
Subcutaneous Infection with Mycobacterium abscessus in a Renal Transplant Recipient

B.M. Prinz, S. Michaelis,

N. Kettelhack, B. Mueller, G. Burg,

W. Kempf . . . . . . . . . . . . . 259

Purpura fulminans in a Child as a Complication of Chickenpox Infection

A. Campanelli, G. Kaya, A.H. Ozsahin,

G. La Scala, C. Jacquier, M. Stauffer,

F. Boehlen, P. de Moerloose,

J.-H. Saurat . . . . . . . . . 262

Pancreatic Panniculitis in a Patient with an Acinar Cell Cystadenocarcinoma of the Pancreas

H.S. Beltraminelli, S.A. Buechner,

P. Häusermann . . . . . . . . 265

Cutaneous Myiasis due to

\section{Dermatobia hominis}

E.J. Hohenstein, S.A. Buechner . . . 268

\section{Giant Fibrokeratoma of the}

Heel

C. Bron, B. Noël, R.G. Panizzon ～. . . 271

Localized Childhood Vulval

Pemphigoid Treated with

Tacrolimus Ointment

S. Lebeau, C. Mainetti, I. Masouyé,

J.-H. Saurat, L. Borradori . . . . . . 273
Lupus-Like Syndrome

Associated with Statin Therapy

B. Noël, R.G. Panizzon . . . . . . . 27

Nephrogenic Fibrosing

Dermopathy Treated with

Extracorporeal Photopheresis

S. Läuchli, C. Zortea-Caflisch,

F.O. Nestle, G. Burg, W. Kempf _ . . 278

Familial Cutaneous Mycosis fungoides: Successful

Treatment with a Combination of Gemcitabine and

Alemtuzumab

P. Weder, M. Anliker, P. Itin,

M. Bargetzi . . . . . . . . . . . . . . 281

Fulminant Herpetic Sycosis:

Atypical Presentation of

Primary Herpetic Infection

A. Campanelli, G. Marazza, L. Stucki,

S. Abraham, C. Prins, G. Kaya,

V. Piguet, J.-H. Saurat . . . . . . . . 284

Cutaneous Plasmablastic Lymphoma in an HIV-Positive Male: An Unrecognized Cutaneous Manifestation

P. Häusermann, N. Khanna, M. Buess, P.H. Itin, M. Battegay, S. Dirnhofer,

S.A. Buechner . . . . . . . . . . 287 